\title{
CONOCIMIENTOS FÍSICOS EN ESTUDIANTES ECUATORIANOS EGRESADOS DE LA EDUCACIÓN BÁSICA SUPERIOR
}

\author{
PHYSICAL KNOWLEDGE IN ECUADORIAN STUDENTS FROM \\ UPPER BASIC EDUCATION
}

\author{
Luis Enrique Hernández Amaro \\ Universidad Nacional de Educación. UNAE. Ecuador. \\ luis.hernandez@unae.edu.ec \\ Ana Mari Pimentel Garriga \\ Universidad Nacional de Educación. UNAE. Ecuador. \\ ana.pimentel@unae.edu.ec
}

\section{RESUMEN}

Las investigaciones sobre las concepciones alternativas de los alumnos, ante muchos fenómenos naturales, así como sus causas y características demuestran que después de muchos años de escolaridad, incluso más de una década, en los que se ha intentado enseñar a los alumnos las concepciones correctas, estos continúan teniendo concepciones alternativas que no se corresponden con las científicamente aceptadas. El presente trabajo se ha elaborado con el objetivo de valorar la existencia de concepciones alternativas sobre la Física en estudiantes ecuatorianos egresados de la Educación Básica Superior en la provincia de Cañar en Ecuador. Para ello se proyectó una investigación con un alcance exploratorio y una metodología cuantitativa de investigación. Para la obtención de información se utiliza una prueba de selección múltiple elaborada a partir de un análisis exhaustivo en el currículum de Ciencias para la Educación Básica Superior y la revisión minuciosa a otras pruebas que han sido aplicadas con estos mismos fines en otras investigaciones. Los resultados se analizaron e interpretaron mediante la construcción de las tablas de frecuencia para cada uno de los ítems, evidenciando la existencia de ideas alternativas acerca de la Física entre los egresados del Educación Básica Superior en la provincia de Cañar en Ecuador.

Palabras clave: Concepciones alternativas, estudiantes de la Educación Básica Superior, enseñanza de las ciencias

\begin{abstract}
Research on alternative conceptions of students, in the face of many natural phenomena, as well as their causes and characteristics, shows that after many years of schooling, even more than a decade, in which students have been taught the correct conceptions, these continue to have alternative conceptions that do not correspond to the scientifically accepted ones. The present work has been developed with the objective of evaluating the existence of alternative conceptions about science in Ecuadorian students graduated from the Higher Basic Education in the province of Cañar in Ecuador. For this, a research with an exploratory scope and a quantitative research methodology was projected. To obtain information, a multiple-choice test is used based on an exhaustive analysis in the curriculum of Sciences for Higher Basic Education and a thorough review of other tests that have been applied for these same purposes in other investigations. The results were analyzed and interpreted by constructing the frequency tables for each of the items, evidencing the existence of alternative ideas about science among the graduates of Higher Basic Education in the province of Cañar in Ecuador.
\end{abstract}

Keywords: Alternative conceptions, students of the Higher Basic Education, science teaching

Recibido: 21 de noviembre de 2016

Aprobado: 15 de abril de 2017

Publicado: 30 de julio de 2017 


\section{INTRODUCCIÓN}

El desarrollo de la sociedad ecuatoriana reclama de constantes cambios y transformaciones a favor de la formación del hombre, como principal protagonista de dicho desarrollo, por lo que es imprescindible la preparación de profesionales, cuyo encargo social está dirigido precisamente a la formación de las nuevas generaciones.

En los momentos actuales se hace más evidente que el desarrollo científico, tecnológico y social en Ecuador está determinado, en gran medida, por la calidad de la preparación general, politécnica y laboral que reciban los estudiantes durante su tránsito por la enseñanza general básica y el bachillerato, lo cual le permitirá posteriormente, asumir con espíritu activo y creador, la solución de las diversas tareas que demanda el desarrollo económico del país.

Vivimos en tiempos en los que la tendencia del desarrollo histórico contemporáneo está marcado por el proceso de globalización: fenómeno de carácter objetivo, resultante del extraordinario desarrollo de la ciencia, la técnica, las comunicaciones y las fuerzas productivas en general. Es por ello que resulta de vital importancia dar prioridad a la inversión en educación, la ciencia y la tecnología, así como la aplicación de los resultados de la investigación científica en el proceso productivo, independientemente de las dificultades que hoy enfrenta el país.

La Conferencia Mundial sobre la Ciencia para el siglo XXI, auspiciada por la UNESCO y el Consejo Internacional para la Ciencia, declara:

Para que un país esté en condiciones de atender a las necesidades fundamentales de su población, la enseñanza de las ciencias y la tecnología es un imperativo estratégico. Como parte de esa educación científica y tecnológica, los estudiantes deberían aprender a resolver problemas concretos $y$ a atender a las necesidades de la sociedad, utilizando sus competencias y conocimientos científicos y tecnológicos. (UNESCO, 1999).

Y se añade: "Hoy más que nunca es necesario fomentar y difundir la alfabetización científica en todas las culturas y en todos los sectores de la sociedad,...a fin de mejorar la participación de los ciudadanos en la adopción de decisiones relativas a la aplicaciones de los nuevos conocimientos" (UNESCO, 1999).

Las propuestas actuales a favor de una alfabetización científica, no solo para los estudiantes de los distintos niveles, sino para todos los ciudadanos, van más allá de la tradicional importancia concedida, más verbal que realmente a la educación científica y tecnológica, para hacer posible el desarrollo futuro. Esa educación científica se ha convertido en opinión de los expertos, en una exigencia urgente, en un factor esencial del desarrollo de las personas y de los pueblos, también a corto plazo.

La educación científica aparece así como una necesidad del desarrollo social y personal. Pero las expectativas puestas en la contribución de las ciencias a unas humanidades modernas no se han cumplido y asistimos a un fracaso generalizado $y$, lo que es peor, a un creciente rechazo de los estudiantes hacia el aprendizaje de las ciencias e, incluso, hacia la ciencia misma. (Valdés Castro, 2002)

Esta preocupante distancia entre las expectativas puestas en la contribución de la educación científica a la formación de ciudadanos conscientes de las repercusiones sociales de la ciencia y susceptibles de incorporarse, en un porcentaje significativo, a sus tareas y la realidad de un amplio rechazo de la ciencia y su aprendizaje, ha terminado por dirigir la atención hacia cómo se está llevando a cabo esa educación científica.

Se asume la definición de educación científica brindada en (UNESCO, 2005) la que la considera como:

“(...) un proceso continuo y permanente, orientado a la formación y desarrollo de la cultura científica, que contribuye a preparar al hombre para la vida, fundamentalmente con conocimientos científicos vinculados al desarrollo social, de procedimientos y habilidades necesarios para su desarrollo y autoeducación y valores éticos acordes a las necesidades sociales. Sus finalidades fundamentales son: brindar conocimientos para continuar estudios superiores, despertar el interés y respeto por la Ciencia, preparar al estudiante para su inserción social y laboral.

Los objetivos de la educación científica dirigidos a potenciar la cultura científica son: el aprendizaje de conceptos culturales y la interpretación de modelos, el desarrollo de destrezas cognitivas y de razonamiento científico, el desarrollo de destrezas experimentales y de resolución de problemas, el desarrollo de actitudes y valores, el desarrollo de una rica imagen de las ciencias, el desarrollo de habilidades de comunicación grupal, el desarrollo del espíritu reflexivo, cooperativo y creativo" (p. 11) 
De lo anterior se deduce que una educación científica dirigida a potenciar la cultura científica debe manifestarse en la capacidad que tienen los diferentes aprendizajes de generar sentimientos, actitudes y valores en los estudiantes. Esto no surge como resultado automático de un proceso de enfrentamiento del estudiante con una determinada información o experiencia afectiva, moral o socialmente relevante sino como resultado de un proceso largo que requiere de la implicación activa de los estudiantes hacia las situaciones de aprendizaje a que se enfrentan y de la posibilidad de valorar sus contenidos desde diversos ángulos y perspectivas, de analizar las consecuencias y de relacionar los contenidos con sus propias conductas.

Este análisis de la enseñanza de las ciencias ha mostrado, entre otras cosas, graves distorsiones de la naturaleza de la ciencia que justifican, en gran medida, tanto el fracaso de buen número de estudiantes como su rechazo a dicho campo de estudio. En efecto, numerosas investigaciones han mostrado que la enseñanza transmite visiones de la ciencia que se alejan notoriamente de la forma como se construyen y evolucionan los conocimientos científicos (Fernández, Gil Pérez, \& Valdés, 2004). Visiones empobrecidas y distorsionadas que generan el desinterés, cuando no el rechazo, de muchos estudiantes y se convierten en un obstáculo para el aprendizaje.

Ello está relacionado con el hecho de que la enseñanza científica, incluida la universitaria, se ha reducido básicamente a la presentación de conocimientos ya elaborados, sin dar ocasión a los estudiantes de asomarse a las actividades características de la actividad científica, lo que repercute significativamente en las dificultades que afrontan los estudiantes en su aprendizaje. Esas dificultades se extienden también a los propios docentes en ejercicio. A esto se suma, además, la necesidad impostergable de actualizar los cursos, ponerlos en correspondencia, tanto en el contenido como en la forma de trabajo, con los adelantos de la ciencia y la técnica.

Otra de las dificultades se refiere al fracaso en la resolución de problemas. Una parte considerable de la investigación didáctica sobre este aspecto ha estado dirigida a mostrar diferencias entre expertos y principiantes, donde el fracaso se atribuye comúnmente a las carencias de los estudiantes, lo que sin duda constituye una de las ideas claves en el pensamiento del profesorado, que no suele cuestionar, en cambio, la didáctica de la resolución de problemas, ni mucho menos las características del proceso de enseñanza en su conjunto.

También se han detectado dificultades en abordar el trabajo experimental en las clases de Ciencias, donde ha prevalecido un enfoque tradicional o académico de realizar las prácticas de laboratorio de forma que al estudiante se le dice exactamente lo que tiene que hacer, con qué, cómo y qué resultados obtener; por encima del enfoque contrapuesto, denominado investigativo, experimental o de proyecto que se fundamenta en concebir las clases experimentales orientadas a presentar las Ciencias Experimentales como una indagación de la naturaleza.

Según los criterios de (Rodríguez \& DíazHigson, 2012), "Ios estudiantes cuando se disponen a aprender conceptos científicos escolares tienen ideas previas, las cuales les sirven para interpretar lo que se les está enseñando, de modo que las nuevas ideas interaccionan con sus esquemas previos" (p. 2)

Son muchas las investigaciones sobre las concepciones alternativas de los alumnos, ante muchos fenómenos naturales, así como sus causas y características, (Blanco López \& Prieto Ruíz, 1997); (Pozo, 1996); (Furió, 1997); entre otras; mismas que demuestran que después de muchos años de escolaridad incluso más de una década, en los que se ha intentado enseñar a los alumnos las concepciones correctas, estos continúan teniendo concepciones alternativas que no se corresponden con las científicamente aceptadas.

La situación descrita también es aplicable en el contexto del sistema educativo ecuatoriano, específicamente en la provincia de Cañar, ya que al egreso de la Educación Básica Superior, los estudiantes poseen concepciones alternativas que invitan a valorar acerca del papel de la enseñanza de las ciencias en la reconstrucción de los modelos mentales con que los individuos entran a la escuela.

\section{MATERIALES Y MÉTODOS}

La Provincia de Cañar es una de las 24 provincias que conforman la República del Ecuador, situada al sur del país, en la zona geográfica conocida como región interandina o sierra. Su capital administrativa es la ciudad de Azogues, mientras la urbe más grande y poblada es La Troncal. Ocupa un territorio de unos $3.908 \mathrm{~km}^{2}$, siendo la quinta provincia del país más pequeña por extensión, detrás de Bolívar, Tungurahua, Carchi y Santa Elena. Limita al norte con Chimborazo, al sur con 
Azuay, por el occidente con Guayas y al este con Morona Santiago.

En el territorio cañarense habitan 225.184 personas, según el último censo nacional (2010), siendo la décimo quinta provincia más poblada del país. La Provincia de Cañar está constituida por 7 cantones, de las cuales se derivan sus respectivas parroquias urbanas y rurales. Según el último ordenamiento territorial, la provincia de Cañar pertenecerá a una región comprendida también por las provincias de Azuay y Morona Santiago, aunque no esté oficialmente conformada, denominada Región Centro Sur.

En el aspecto agrario existe variedad de cultivos como trigo, cebada, papas, verduras, legumbres, hortalizas y áreas de pastizales en las zonas templadas; en los sectores cálidos se produce café, arroz, caña de azúcar, banano y varias frutas de tipo tropical. En las áreas boscosas se puede encontrar especies tales como cedro, laurel, palo prieto e ishpingo. La ganadería tiene su mayor importancia en la crianza de ganado vacuno, lo que permite una buena producción de leche y carne. La pequeña industria y de manufactura es considerada valiosa fuente de ingresos económicos, principalmente en las ramas alimenticia, del calzado, textil y muebles de madera.

Teniendo en cuenta la información que se desea obtener, la investigación se proyecta con un alcance exploratorio y una metodología cuantitativa de investigación. Para la obtención de información se utiliza una prueba de selección múltiple que permite la aplicación a un gran número de estudiantes en un periodo de tiempo relativamente corto. Para seleccionar dicha prueba se realizó un análisis exhaustivo en el currículum de Ciencias para la Educación Básica Superior (1) y la revisión minuciosa a otras pruebas que han sido aplicadas con estos mismos fines en otras investigaciones (2) y (3); posteriormente se realizó un proceso de valoración de la misma a partir del criterio de docentes con experiencia de las propias instituciones educativas que permitió enriquecerla y constituirla en un instrumento adecuado para la investigación.

De este modo quedó constituida dicha prueba por 30 ítems de los cuales se corresponden con Reposo de los objetos (2), Movimiento horizontal de los objetos (7), Caída de los objetos (6), Trayectoria de los objetos (3), Concepciones sobre energía (7) y Concepciones sobre calor (5).

Los resultados se analizarán e interpretarán mediante la construcción de las tablas de frecuencia para cada uno de los ítems empleando el procesador SPSS versión 23.

De acuerdo con la base de datos disponible en http://educacion.gob.ec/amie/, en la provincia de Cañar, en el mes de diciembre de 2016 había un total de 851 estudiantes cursando el décimo año y último de la Educación General Básica. Esta cantidad se ha dividido en diferentes estratos teniendo en cuenta el tipo de sostenimiento (Fiscal, Fiscomisional 0 Particular) y el tipo de zona (Urbana o rural).

Tabla 1. Resultados del proceso de selección muestral por tipos de instituciones educativas en la provincia de Cañar.

\begin{tabular}{|l|c|c|}
\hline Tipo de institución educativa & $\begin{array}{c}\text { Cantidad de } \\
\text { Estudiantes }\end{array}$ & Muestra \\
\hline Fiscal rural & 17 & 8 \\
\hline Fiscal urbana & 13 & 6 \\
\hline Fiscomisional rural & 0 & 0 \\
\hline Fiscomisional urbana & 484 & 0 \\
\hline Particular rural & 0 & 168 \\
\hline Particular urbana & 337 & 423 \\
\hline Total & 851 & 241 \\
\hline
\end{tabular}


A partir de los datos disponibles se calculó el tamaño estimado de la muestra para un error del $5 \%(0.05)$ con un nivel de confianza del $95 \%$ (0.95) y un valor de $z=1.96$, lo que dio el tamaño necesario de la muestra que resultó ser de 423, posteriormente se calcularon los valores de la muestra en cada uno de ellos de modo que el número de elementos muestrales de cada estrato fuera directamente proporcional al tamaño del estrato dentro de la población (afijación proporcional).

\section{RESULTADOS Y DISCUSIÓN}

Luego de seleccionadas las instituciones educativas y con ayuda de estudiantes de la Universidad Nacional de Educación (UNAE), fueron aplicados los cuestionarios, calificados y organizadas las respuestas.

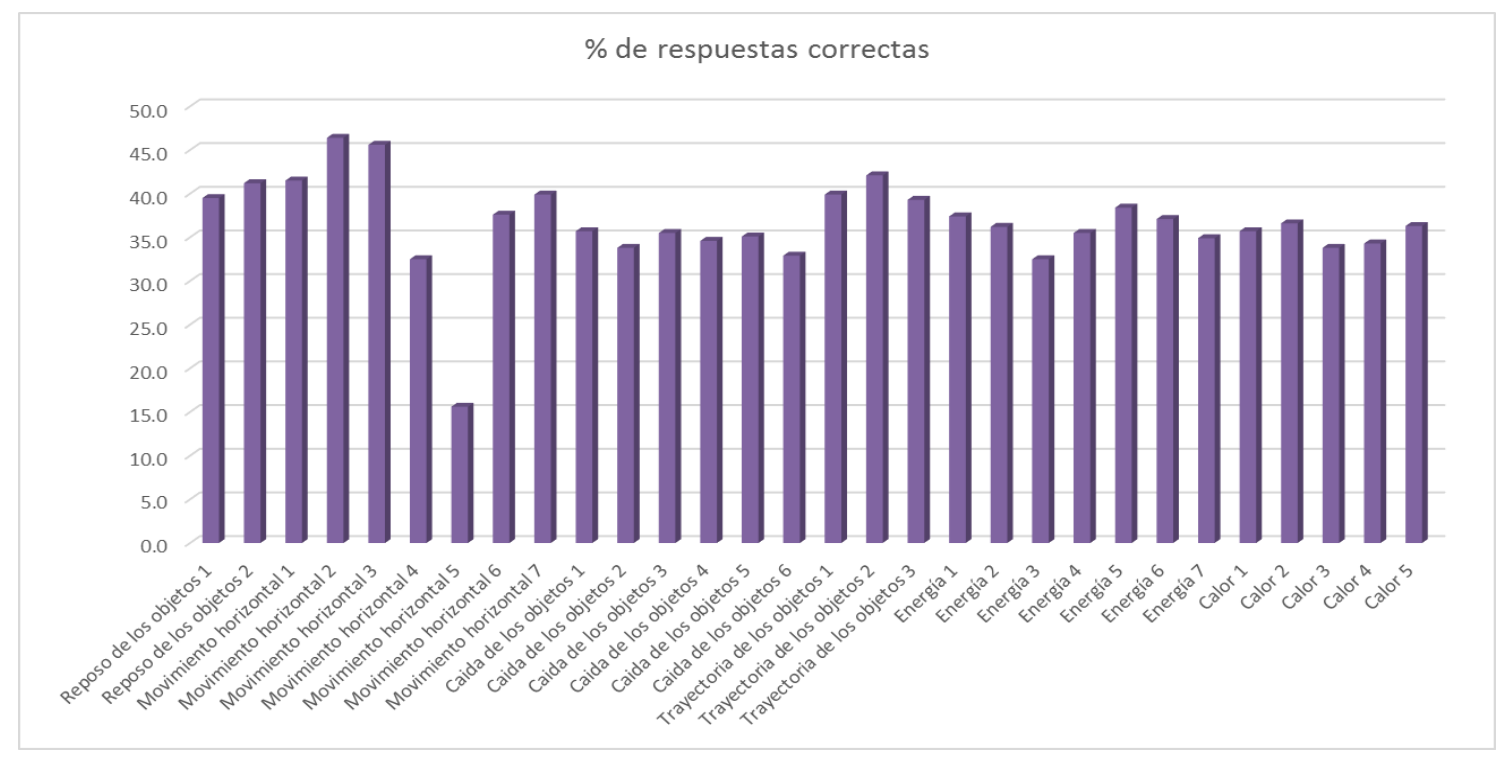

Figura 1. Porcentajes de respuestas correctas en cada uno de los ítems. Elaboración propia.

Las frecuencias de respuestas correctas en los ítems relacionados con el reposo de los objetos son del 39.5 y $41.2 \%$ respectivamente. En el caso del movimiento horizontal de los objetos esta frecuencia alcanza valores de $41.5,46.4$, $45.6,32.5,15.6,37.6$ y $39.9 \%$ en este orden.

Para la caída de los objetos los porcentajes de las frecuencias se encuentran desde 32.9 y hasta 35,7\% de respuestas correctas. Mientras, para las preguntas relacionadas con la trayectoria de los objetos las frecuencias oscilan entre el 39.3 y $42.1 \%$; en tanto en las interrogaciones sobre energía las respuestas correctas fluctúan desde 32.5 y hasta $37.4 \%$.

Las concepciones alternativas de los estudiantes ecuatorianos egresados de la Educación Básica Superior en la provincia de Cañar sobre el concepto de energía, aluden a tres áreas problemáticas: el concepto de energía, los procesos que implican la transferencia de energía y el principio de conservación de la energía, resultado que coincide con estudios realizados por (3).

Para el caso del estudio relacionado con las concepciones alternativas acerca del calor, los ítems aplicados revelaron respuestas correctas entre el 33.8 y $36.6 \%$ de los casos; para estos estudiantes el calor puede entrar o salir y pasar de unos cuerpos a otros, significado este contrario al científico, que es una forma de transferencia de energía, entre dos sistemas que se encuentran a diferente temperatura.

Cabe señalar que dentro de los estudios acerca de la ciencia Física, la definición de conceptos, la explicación de fenómenos y la resolución de ejercicios y problemas asociados al movimiento, la energía y el calor son básicos para entender el resto de contenidos que deben ser comprendidos para su aplicación en la práctica y para entender los procesos básicos de la naturaleza, de ahí la enorme importancia de concientizar al profesorado en este sentido. 


\section{CONCLUSIONES}

La presente investigación de alcance exploratorio, demuestra la existencia de concepciones alternativas en los estudiantes egresados de la Educación Básica Superior en la provincia de Cañar; mismas que están relacionadas con el reposo, movimiento, caída y trayectoria de los objetos así como con la energía y el calor como forma de transferencia.

Así, la investigación se ha ocupado principalmente de evidenciar el fracaso de los estudiantes al enfrentarse a este tipo de situaciones; pero el análisis sugiere ir más allá y preguntarse: ¿en qué medida lo que se enseña en las clases se aproxima a una verdadera solución de problemas? Esta interrogante cuestiona las habituales orientaciones didácticas de profesores y libros de texto y responde a una hipótesis distinta a las anteriores: un fracaso en este sentido puede tener su origen en errores de planteamiento en el proceso de enseñanza aprendizaje, cuestión que será atendida en otras investigaciones

\section{REFERENCIAS}

1. MINEDUC. Ministerio de Educación. Ecuador. [Online].; 2016. Available from: http://educacion.gob.ec/wpcontent/uploads/downloads/2016/03/CCN N1.pdf.

2. Camargo ÉPd, Scalvi LVdA, Braga TMS. CONCEPCIONES ALTERNATIVAS SOBRE REPOSO Y MOVIMIENTO, MODELOS HISTÓRICOS $Y$ DEFICIENCIA VISUAL. ENSEÑANZA DE LAS CIENCIAS. 2007;: p. 171-182.

3. Rodríguez V, Díaz-Higson S. CONCEPCIONES ALTERNATIVAS SOBRE LOS CONCEPTOS DE ENERGÍA, CALOR Y TEMPERATURA DE LOS DOCENTES EN FORMACIÓN DEL INSTITUTO PEDAGÓGICO EN SANTIAGO, PANAMÁ. Actualidades investigativas en educación. Universidad de Costa Rica. 2012;: p. 1-26.

4. Valdés Castro $P$. Enseñanza de la Física Elemental La Habana. Cuba: Pueblo y Educación; 2002.

5. Fernández I, Gil Pérez D, Valdés P. La superación de las visiones deformadas de la ciencia y la tecnología: un requisito esencial para la renovación de la educación científica La Habana. Cuba: Pueblo y Educación; 2004.

6. UNESCO. ¿Cómo promover el interés por la cultura científica? Una propuesta didáctica fundamentada para la educación científica. In ; 2005; Santiago de Chile: ORELAC/UNESCO. p. 11.

7. Alonso Vázquez A. Concepciones alternativas en Física y Química de Bachillerato: una metodología diagnóstica. Enseñanza de las Ciencias. 1990;: p. 251258.

8. Pozo J. Las ideas del alumnado sobre la ciencia: de dónde vienen, a donde van.y mientras tanto qué hacemos con ellas. Alambique: Didáctica de las ciencias experimentales, 7. 1996;: p. 18-26.

9. Furió C. Las concepciones alternativas del alumnado en ciencias: dos décadas de investigación. Resultados y tendencias. Alambique: Didáctica de las ciencias experimentales $7.1997 ;:$ p. 7-17.

10 Blanco López Á, Prieto Ruíz T. Las concepciones de los alumnos y la investigación en Didáctica de las Ciencias. Málaga:; 1997.

11 UNESCO. DECLARACION SOBRE LA CIENCIA Y EL USO DEL SABER CIENTIFICO. In ; 1999; Budapest. Hungría. 